\title{
NEUROLEPTIC MALIGNANT SYNDROME IN CHILDREN AND ADOLESCENTS: SYSTEMATIC REVIEW OF CASE REPORTS.
}

\section{León-Amenero, Deborah'1. Huarcaya-Victoria, Jeff ${ }^{1,2}$. \\ Departamento de Psiquiatría, Hospital Nacional Guillermo Almenara Irigoyen. EsSalud. Lima - Perú. \\ Universidad de San Martín de Porres, Facultad de Medicina, Centro de Investigación en Salud Pública. Lima, Perú.}

\section{INTRODUCTION}

The neuroleptic malignant syndrome (NMS) is a rare and potentially fatal drug adverse reaction, commonly associated with antipsychotic (neuroleptic) medications, but not limited to these (1). There are still few studies of this entity in the child and adolescent population. Apparently, it is postulated that the course and treatment in these are usually the same as in adults, however, more studies are needed $(2,3)$.

\section{OBJECTIVES}

To describe the clinical, laboratory and treatment characteristics of children and adolescents with NMS and factor structure of the symptoms.

\section{MATERIAL AND METHODS}

The guidelines of the PRISMA Statement guide were followed (Figure 1). A MEDLINE / PubMed search of all reported cases of NMS from January 2000 to November 2018 was performed and demographic, clinical, laboratory and therapeutic variables were identified. Descriptive statistics of the cases were used. A factor analysis of the symptoms was performed.

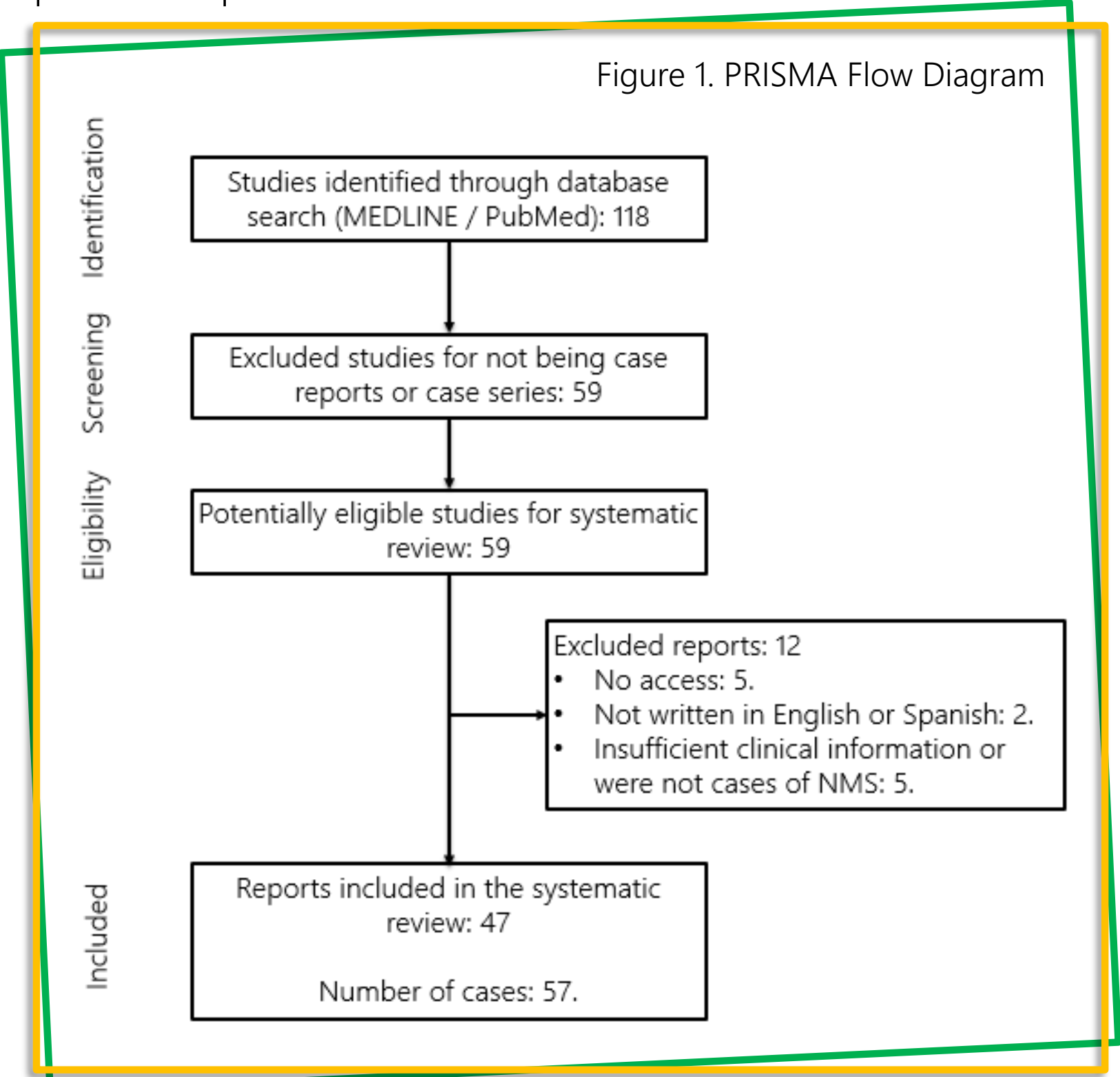

RESULTS

The sample included 57 patients (42 men and 15 women), with a mean age of 13.65 years $( \pm 3.89$ ). The most frequent diagnosis was acute psychotic disorder (21.1\%) and the onset of the NMS occurred at $11.25 \pm$ 20.27 days (typical antipsychotics) and at $13.69 \pm 22.43$ days (atypical antipsychotics). The most frequent symptoms were muscle rigidity (84.2\%), autonomic instability (84.2\%), fever (78.9\%) and impaired consciousness (73.7\%) (Graphic 1). No significant differences were found between men and women in the frequency of symptoms.

Graphic 1. FREQUENCY OF SYMPTOMS

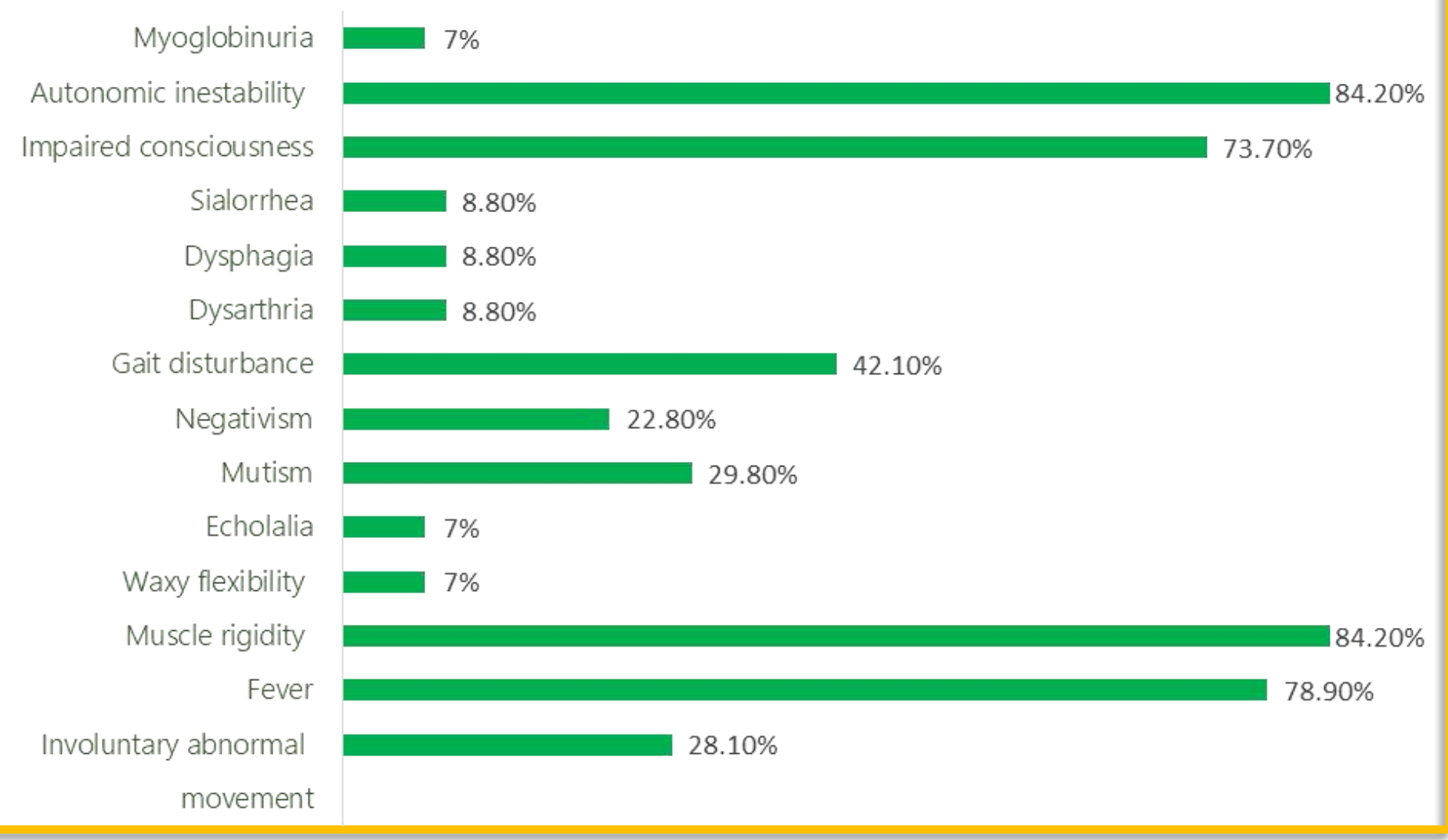

The most frequent laboratory findings were CPK elevation (100 IU/I) with a mean of 84.30 ( \pm 214.53) and leukocytosis (42.1\%). Regarding treatment, the most frequent measure was the use of benzodiazepines (28.1\%) followed by support measures (24.6\%).

\section{FACTORIAL ANALYSIS OF THE SYMPTOMS}

Three factors were identified: 1) "Catatonic" with mutism (0.912), negativism (0.825) and waxy flexibility (0.522); 2) "Extrapyramidal syndrome" with gait disturbance (0.860), involuntary abnormal movements (0.605), muscle rigidity (0.534) and sialorrhea (0.430); 3) "Autonomic instability" with fever (0.798), impaired consciousness (0.795) and autonomic instability (0.387).

\section{CONCLUSIONS}

The NMS in children and adolescents was more frequent in men and had a mean onset time of 12.51 days. According to the factorial analysis of the symptoms, the NMS could be of three types: catatonic, extrapyramidal and autonomic unstable. Finally, the NMS is still unpredictable, infrequent and does not have specific clinical markers, for what it requires more studies.

Pileggi DJ, Cook AM. Neuroleptic Malignant Syndrome. Ann Pharmacother. 2016;50(11):973-81.

Neuhut R LJ, Silva R. Neuroleptic Malignant Syndrome in Children and Adolescents on Atypical Antipsychotic Medication: A Review. J Child Adolesc Psychopharmacol. 2009;19(4):415-22. 